\title{
LA FILOSOFÍA DE LA CIENCIA CONTABLE
}

The philosophy of science acconting

\author{
Arturo Torres Gallardo * \\ Lima-Perú \\ [Recepción: Setiembre de 2012/ Conformidad: Octubre de 2012]
}

\section{RESUMEN}

La contabilidad como cualquier otra disciplina moldea y delimita la realidad al construir su objeto de estudio, a través de un proceso lento pero creciente en el que coexisten diversas posturas ideológicas, las cuales le atribuyen objetos de estudio diferentes, pero lo que ellas tienen en común es que han estado alrededor de la cuestión patrimonial o la riqueza del ente económico, que representa su realidad económica. El conocimiento contable es racional al formular juicios y razonamientos coherentes entre sus enunciados. Sin embargo, atendiendo a los principios y no al desarrollo formal de las ciencias, los cuales pueden racionales o experimentales, sostengo que es una ciencia experimental. La disciplina contable es una ciencia aplicada y en proceso de maduración, debido a que no existe un paradigma predominante, sino es una ciencia multi-paradigmática. Esta propuesta está fundamentada en evidenciar dentro de la disciplina contable la presencia de tres elementos: El objeto de estudio: El patrimonio del ente económico y su dinámica; el método: inductivo-deductivo y, la capacidad de predicción, expresada en los modelos contables existentes los cuales se evidencian. Por tanto, bajo esta modesta perspectiva, la disciplina contable posee un objeto de estudio, un método y capacidad de predicción, lo que le confiere un estatus de ciencia.

Palabras Clave: Disciplina, Ciencia, Elementos de la ciencia, Objeto de estudio, Método, Capacidad de Predicción

\begin{abstract}
The accounting knowledge is rational in rendering coherent judgments and arguments, between their statements. However, according to the principles, and not to the formal development of the sciences, which can be rational or experimental, I argue that it is an experimental science. The accounting discipline is an applied science and it is in a maturation process, because of there is no dominant paradigm, but a multi-paradigmatic science.

This proposal is based in found inside the accounting discipline the presence of three elements: the study object (the assets of the economic entity and its dynamics), the method (inductive-deductive), and predictive capacity, expressed in existing accounting models, which are evident. Therefore, under this modest perspective, the accounting discipline has an object of study, a method and predictive capacity, which gives it a status of science.
\end{abstract}

Keywords: discipline, science, elements of science under study, method, predictive capacity.

* Egresado de los Programas de Doctorado en: Ciencias Económicas y Sociales en la Universidad Johannes Kepler, Linz-Austria; y de Contabilidad y Finanzas de la Escuela de Post Grado de la Universidad Nacional de Trujillo, Perú. Email:atorresg42@hotmail.com 


\section{INTRODUCCIÓN}

La disciplina contable se origina en un saber milenario. Los primeros aportes sobre esta disciplina fueron hechos por Luca Pacioli (1494) en su obra: Summa de arithmetica, geometría proportioni et proportionalita. Desde el principio tuvo un crecimiento teórico y metodológico importante, pero su consideración dentro del campo de las ciencias se da a partir de la segunda mitad del siglo XX. Este proceso se inicia al transferir los métodos e instrumentos de las ciencias duras al estudio de la contabilidad. Los primeros aportes fueron proporcionados por Cerboni (1886), Besta (1909), Masi (1927).

Un concepto fundamental introducido en la filosofía de la ciencia, y en la ciencia contable bajo la mirada kuhniana es el término paradigma, el cual no tiene una definición unívoca (Barker, 1995). La sistematización de Kuhn es la más aceptada por los críticos de la sociología de la ciencia (Montes Salazar y otros, 2006). Destacan entre estos el Trabajo de Belkaoui (1991), cuyo título es: La contabilidad una ciencia multi-paradigmatica, el cual nos permite afirmar que la disciplina contable está formada por varios paradigmas, entre ellos: el funcionalista, el interpretativo, el radical humanista y el radical estructuralista.

Bajo la perspectiva Kuhniana, la característica que distingue a la ciencia de la no ciencia es la existencia de un paradigma capaz de apoyar una tradición de ciencia normal o madura. El paradigma establece las normas necesarias para legitimar el trabajo dentro de la ciencia que rige. Coordina y dirige la actividad de "resolver problemas" que efectúan los científicos normales que trabajan dentro de una ciencia madura (Chalmers, 1984). Des- de una visión sociológica y no filosófica, "el paradigma Kuhniano es un conjunto de hábitos de los científicos, ya sean intelectuales, verbales, tecnológicos, de comportamiento, etc." (Wirth, 2001), o también podría definirse como "Un conjunto de instrumentos conceptuales que configuran una determinada manera de hacer ciencia y de administrar un conjunto de logros científicos, desde el contexto genético hasta el de la praxis" (Tua Pereda, 1983).

\subsection{Que es una disciplina}

El término disciplina es "consecuencia del fraccionamiento de la realidad en parcelas del conocimiento" (Gómez Villegas, 2003). Fue un esquema propiciado por la dinámica de la Universidad en el siglo XIX con el auspicio del proyecto científico de la modernidad y de la dinámica propia de la división del trabajo, en este caso el intelectual, para lograr niveles de especialización que permitieran el desciframiento de la realidad a partir de la des-encriptación y representación de la vida natural y social (Morín, 1998; Pág. 8).

La disciplina es la sistematización de conocimientos fruto de la sociedad y al servicio de esta, para el bienestar de sus miembros. El propósito de la disciplina es comprender las partes y elementos que constituyen al hombre en tanto unidad biológica y miembro social, en tanto elemento de un complejo sistema del que es parte, no su totalidad.

Las disciplinas científicas se caracterizan, según la perspectiva epistemológica clási$\mathrm{ca}^{1}$, por la identificación de unos elementos propios mínimos: el Objeto sobre el que la forma de conocimiento trata; el método por el cual es abordado tal objeto de la realidad; las teorías que se construyen a partir del paulatino descubrimiento de la misma realidad; y el sujeto mismo que conoce.

1 Nos referimos aquí a la filosofía de las ciencias y del conocimiento, desde Aristóteles, pasando por descartes y llegando a los empiristas y positivistas. En Briones Guillermo. La ciencia y la modernidad. Editorial Ariel. México 1964. 


\subsection{La realidad actual de la disciplina contable}

Los últimos cinco siglos de la contabilidad muestran un desarrollo de la disciplina sustentada en procesos de acumulación, situación que lleva a que ciertas corrientes contables afirmen que la contabilidad no ha tenido una verdadera revolución científica. Al parecer, los profesionales-técnicos no perciben anomalías en su proceso de representación de la realidad (ejemplo: cifras frías registradas sin contexto, costos ambientales, etc.), llegando a confundir la exactitud de la ciencia instrumental (matemática) que aplican, con la exactitud de la propia contabilidad.

La realidad socio-económica actual viene formulando nuevas exigencias en materia contable. Ello significa que, el sistema contable actual basado en lo financiero es insuficiente para resolver las demandas actuales en los aspectos ambientales, los sociales y culturales. En opinión de Viegas (2003) ${ }^{2}$, la contabilidad se encuentra en crisis caracterizada por una pérdida de confianza de los usuarios externos debido a las malas prácticas y la falta de investigación en contabilidad. Montes $y$ otros (2006) mencionan que frente a esta situación se presentan dos opciones:

1) Que el sistema contable económicofinanciero actual pueda a partir de una ampliación de sus funciones y objetivos, dar respuestas a las nuevas exigencias de la sociedad. Por ejemplo, el proceso de regulación contable que actualmente atraviesa por una gran dinámica.

2) Que el modelo contable actual no tenga la capacidad de orientarse hacia esos nuevos fines, y se requiera un cambio de estructura fuerte genere una verdadera revolución científica alrededor de la disciplina.

Existen opiniones que no niegan la importancia de la regulación contable y su relación con la internacionalización de la disciplina contable, señalándose que no ha contado con el suficiente estudio y rigor conceptual. De la misma manera, la estructura conceptual y teoría abstracta de la contabilidad siguen inamovibles, en las "características universales comunes" de todos los sistemas contables no han surgido ninguna transformación en cuanto a transacciones, entidades, valores monetarios, agentes y objetos económicos, sistemas de valoración, etc. (Mattesich, 1964-1995; Cuadrado y Valmayor, 1999).

Se está consciente también que cambios cualitativos y cuantitativos en la ciencia pueden provenir de estudios rigurosos al interior de los paradigmas dominantes, identificando de esta manera sus anomalías.

\subsection{Influencia de la corriente positivista en la contabilidad}

Stephens ${ }^{3}$ (1735) a través de su libro "Italian Book-Keeping reduced into an Art." establece la ecuación patrimonial como una traducción matemática, la que a su vez fue conocida en Inglaterra como la Teoría Patrimonial Cuantitativa o Materialista (Boter 1959) ${ }^{4}$, reduciendo todo el conocimiento a una expresión matemática (a esto se le conoce como el reduccionismo de la contabilidad): Activo = Pasivo + Patrimonio. $\mathrm{Al}$ parecer las cifras producto de las ecuaciones contables han dejado de responder a las exigencias de

2 Viegas, Juan Carlos (2003). “Contabilidad en crisis ¿ciencia o técnica?. Revista Integral de Legis de Contabilidad y Auditoría, p. 160.

3 Citado por Casal y Viloria. La ciencia contable, su historia, filosofía, evolución y su producto. Actualidad Contable FACES, Venezuela. Año 10, N 15, Julio-Diciembre 2007.

4 Idem 3. 
la sociedad. El equilibrio mostrado a través de la ecuación contable, no está reflejando la dinámica existente en las entidades, ni su contexto.

El conocimiento contable está inserto dentro de la filosofía positivista hasta el momento, puesto que estudia los hechos económicos dados por el entorno (sociedad); para proporcionar información y revisar estos hechos o fenómenos (Casal y Viloria, 2002). La contabilidad encuentra en el positivismo un espacio que considera propio, y tanto es así, que el ejercicio de la profesión ha estado recargado de cuantificación y determinismo, según lo cual la explicación o el origen del hecho económico, el entorno donde se produce no interesan sólo importa la anotación que mantenga el equilibrio en la ecuación contable. Esta "parálisis paradigmática" (Zaa, 1966), ha impedido desarrollar la disciplina contable como una verdadera ciencia. La ciencia contable debe redefinirse para responder a las nuevas exigencias del entorno, para dejar de presentar cifras frías sin contexto $\mathrm{y}$ en su lugar, tomar una posición frente a la necesidad de registrar y valorar los recursos naturales, cuantificar los costos sociales de la educación o la salud, etc.

\subsection{La tesis de Kuhn y la contabilidad}

Las tesis de Kuhn (1971) han sido aplicadas para estudiar el proceso de desarrollo científico de la Contabilidad y también para analizar la situación actual de la misma (Gomez, s/f), su aplicación pudiera ser cuestionada por las características del entorno económico y social de la contabilidad, el cual ejerce influencia decisiva en su evolución. En esta línea sobresalen los trabajos de Wells (1976) y Belkaoui (1985), y el documento Statement of Accounting Theory and Theory Aceptance (SOATATA) de la American Accounting Association (A.A.A.).
Según Wells (1976) la Contabilidad evoluciona mediante un proceso continuado de revoluciones y de sustitución de paradigmas. Apoyándose en el sentido amplio de paradigma como "matriz disciplinar", Wells trata de descubrir -de acuerdo con las tesis de Kuhnsus elementos observando la conducta de los miembros de la comunidad científica. Aunque la opinión de "comunidad científica" es criticada por Donoso (1995) y Requena (1989); sin embargo, no disminuyen el valor de la propuesta de Wells".

Es pertinente destacar aquí la opinión de Mattessich (1985) cuando señala que, "más que un cambio paradigmático en Contabilidad nos enfrentamos con un cambio metodológico que puede conducir a una perspectiva, que posibilite y solvente cuestiones no planteadas".

\subsection{La concepción multi-paradigmática de Belkaoui}

Según Belkaoui (1985) un paradigma es una imagen esencial de la materia subjetiva de una ciencia, que sirve para definir lo que debiera ser utilizado, las preguntas que hay que hacerse y las reglas que deben seguirse para interpretar las respuestas obtenidas. En definitiva, se trata de la unidad mayor de consenso en una ciencia y sirve para diferenciar una comunidad científica de otra. Este autor somete a análisis la situación actual de nuestra ciencia tomando como referencia los diferentes enfoques de la misma contenidos en el informe "Statement of Accounting Theory and Theory Aceptance" (SOATATA) de la American Accounting Association (AAA), sobre la base de la noción de paradigma $\mathrm{Ku}$ hniano.

Belkaoui señala la existencia de una multiplicidad de paradigmas, que se encuentran en pugna por lograr la máxima aceptación en la comunidad contable: a) Paradigma antro- 
pológico-inductivo, b) Paradigma del beneficio verdadero-deductivo, c) Paradigma de utilidad de la decisión-modelo de decisión, d) Paradigma de utilidad en la decisión-decisor-comportamiento agregado del mercado, e) Paradigma de utilidad en la decisión-decisor-usuario individual, y f) Paradigma de economía de la información. Sobre estos paradigmas se precisa que los dos primeros son los más representativos y los que mejor definen a las principales posiciones contrapuestas de la comunidad científica. El primero se fundamenta en la utilización del método inductivo, mientras que los demás utilizan una metodología deductiva y normativa (Donoso, 1995).

\subsection{Las diversas definiciones atribuidas a la disciplina contable.}

La contabilidad ha sido considerada como una ciencia social (Garcia, 2001; Goxens, 1970; Muñoz, 2003). Ha sido definida también como ciencia empírica (Lisdero, 1972, Gonzalo, 1983); como ciencia que trata sobre los métodos de registración de las transacciones en los negocios (Paton y Stevenson, 1917; Bertora, 1975); como ciencia económica (Mallo Rodriguez, 1991; Cañibano, 1971); como una rama de las matemáticas (Justo y Reyes, 1947); como una ciencia experimental (Millan, 1992). La conceptualización de ciencia aplicada es compartida por Wirth ${ }^{5}$ (1999) quien señala que "la importancia de una ciencia aplicada está dada en la medida que ésta se nutre del conocimiento científico que puede proveer una ciencia pura. La esencia de una ciencia aplicada se encuentra en el uso del conocimiento científico, provisto por una ciencia pura, con un objetivo pragmático", etc. esta diferencia entre ciencia pura y ciencia aplicada radica en que la primera busca la relación causa-efecto, la segunda se orienta a la relación medios-fines (Matessich, 1995, p. 86). Como puede advertirse, las definiciones de contabilidad son diversas y van desde la consideración de ciencia, ciencia en progreso, hasta ser calificada como no ciencia.

Entre las más importantes definiciones tenemos: Fabio Besta (1909), sobre la contabilidad dice: "La contabilidad en su aspecto teórico estudia y enuncia las leyes del control interno económico de las haciendas (empresas) de toda especie y ofrece normas oportunas a seguir para que dicho control pueda resultar realmente eficaz, satisfactorio y completo; en su aspecto práctico es la ordenada aplicación de esas normas -leyes". Vincenzo Masi $(1927)^{6}$ en su obra "La Contabilidad como ciencia", considera que la contabilidad es la ciencia del patrimonio, que tiene por objeto el estudio del patrimonio de la hacienda (empresa) considerado en su aspecto estático y dinámico, cualitativo y cuantitativo. Esta definición es muy peculiar por cuanto contempla un objeto de estudio es sus aspectos estático y dinámico, extendiéndolo hasta el aspecto cualitativo.

Mattessich (1958) quien en opinión de Millán, es considerado como el investigador que más ha estudiado la disciplina contable, ha realizado aportes no sólo desde un punto de vista sintáctico o formal, sino también a nivel semántico y pragmático, define la contabilidad señalando: "la contabilidad es una disciplina que se refiere a las descripciones cuantitativas y predicciones de la circulación de la renta y los agregados de riqueza por medio de un método basado en el conjunto de supuestos básicos: valores monetarios, inter-

5 WIRTH (1999). La realidad de la Contabilidad. Tesis doctoral.

6 Citado Por Millán Puentes: La contabilidad como ciencia, p. 106. 
valos de tiempo, estructura, dualidad, agregación, objetos económicos, descompensación de las obligaciones monetarias, agentes económicos, unidades económicas, transacciones económicas, valoración, realización, clasificación datos de entrada, duración, extensión (consolidación) importancia cuantitativa (materialidad) y distribución". Particularmente, esta definición a nuestro juicio es muy importante por cuanto no ofrece dudas respecto a objeto y métodos, ya que incluye en ella los supuestos básicos precisos para el desarrollo del método. Además, explicita el aspecto predictivo de la información contable, aspecto que contemplamos en nuestra perspectiva de estudio, como un elemento necesario para toda disciplina que posee el estatus de ciencia.

Calafell Castillo (1970), en una forma más omnicomprensiva define la contabilidad: "es una ciencia de naturaleza económica, cuyo objeto de estudio lo constituye la varia realidad económica, no como realidad en sí, sino en su aspecto de conocimiento, tanto cualitativo como cuantitativo, mediante métodos apropiados, con el fin de poner de relieve dicha realidad económica de la manera más exacta posible y de forma que nos muestre cuantos aspectos de la misma interesen”.

Mario Biondi, por su parte, define a la contabilidad como una ciencia empírica orientada hacia una realidad cambiante, procura estudiar científicamente esa realidad, aprehenderla y dirigirla, con un procedimiento similar al de la Economía y la Sociología. Ballestero (1983), señala que la "contabilidad es una ciencia instrumental que se ocupa de sistematizar las técnicas contables y de explicarlas, ya axiomáticamente, ya mediante modelos, según su naturaleza”. Millán (1992) al referirse a la autonomía de la disciplina contable señala "Es una ciencia que desde un principio ha tenido un campo de acción propio y que, aunque tiene contactos o relaciones con otras ciencias, nunca ha pedido prestado ni el objeto, ni los métodos de investigación, ni ha solicitado ayudas doctrinales, ni mucho menos se le ha condenado a una especie de superesclavitud, apartándola del grupo de grupo de la ciencias libres y autónomas".

La contabilidad también ha sido conceptualizada como una construcción social (la acción humana y los hechos sociales). Como tal, proviene del devenir histórico y de las acciones de individuos, que sólo se individúan por medio de la colectividad (Marx, 1976; Morín, 1995). La propuesta de Morín constituye un cambio de paradigma y ha sido denominado el paradigma perdido o de la complejidad y nace en respuesta a una ciencia que ha venido actuando en forma ciega, metódica, aislada y sin control. Es decir, una ciencia determinista, mecanicista, cuantitativa, que ignora, disuelve todo lo subjetivo, afectivo, libre y creador de los fenómenos estudiados, que deja aún lado la multidimensionalidad e interrelación de los factores que inciden en el estudio de estos, arrastrándola a la ineficiencia para proveer respuestas frente a fenómenos que son cada vez más complejos.

Una de las más recientes definiciones de Contabilidad, formulada desde una perspectiva social; es la proporcionada por García Casella (1997, 2001a, 15; 2002, 201; 2009, 57) quien define y fundamenta la cientificidad de la contabilidad al definirla del modo siguiente: "ciencia social, factual, cultural y aplicada que se ocupa de explicar y normar las tareas de descripción, principalmente cuantitativas, de la existencia y circulación de objetos, hechos y personas diversas en cada ente $u$ organismo social y de la proyección de los mismos, en vista al cumplimiento de metas organizacionales, a través de sistemas basados en un conjunto de supuestos básicos". 


\section{Los Elementos de la Ciencia Contable}

$\mathrm{Al}$ ingresar en este campo de la Filosofía de la Ciencia Contable se identifica una polémica. De acuerdo con Mardones (1991) esta polémica se refiere a que no existe un consenso sobre el estatuto de cientificidad de la contabilidad, precisando que ello se origina desde la aparición de las diversas disciplinas que se acogen a la categoría de ciencias económicas y administrativas. Es por ello que, a partir del siglo XIX en adelante que los investigadores buscaron un método y objeto contable, habiendo expresado opiniones diversas, conforme a sus ideologías propias que interpretaron desde su punto de vista la "gnose" contable.

\section{¿Cuál es el objeto de estudio de la discipli- na contable?}

La contabilidad como cualquier otro campo disciplinar moldea y delimita la realidad al construir su objeto de estudio, obteniendo una imagen, una forma de representación especifica de concepción del mundo. Ello se realiza a través de un proceso lento pero creciente, en el que no se aprecia uniformidad y homogeneidad y, por el contrario coexisten teóricos con diversas posturas ideológicas. Sin embargo, el origen primigenio de la problematización contable, estuvo vinculada a estructuras propias del conocimiento lógicomatemático, es así que las primeras manifestaciones de nuestra disciplina se adscribieron ha ámbitos próximos a las matemáticas $y$, en especial a la aritmética comercial (Tua Pereda, 1995).

Existen diversos enfoques sobre el objeto de investigación contable, entre estos los enfoques racionalista, positivista y el últimamente el social. Hay también varias teorías que conforman la disciplina contable, entre ellas: la del propietario, del ente (o su exten- sión llamada teoría del emprendimiento), del accionista mayoritario, del fondo, del control (De Andrade, 2006), utilitaria, patrimonialista, etc. Sobre el particular, Chaves da Silva (2007), menciona las diversas fases de la doctrina y tesis contables que atribuyen a la contabilidad, objetos diferentes de aquellos consagrados actualmente.

La más importante corriente contemporánea del pensamiento filosófico-científico de la Contabilidad es la concepción Neopatrimonialista, cuyo origen se ubica específicamente en Brasil, durante la segunda mitad del siglo XX y se debe su construcción y desarrollo a los esfuerzo científicos del Doctor Antonio

Lopes de Sá, y a los trabajos de sus seguidores en América y Europa (Zaá, 2012) ${ }^{7}$. Conforme a esta teoría, la Contabilidad no debe reducirse solo al estudio de hechos ex post facto aislados, sino que debería dedicarse al conocimiento de las causas de los sucesos, apoyándose en métodos científicos, para luego derivar modelos que permitan acercar la teoría a sus aplicaciones prácticas. Esta teoría enmarca su discurso ontológico en la "célula social", como lo denomina su autor, donde se encuentran las causas primeras de la transformación patrimonial y sus consecuencias, sentando así las bases de una visión holística del método y del ámbito de la investigación científica en Contabilidad.

El objeto de estudio de la contabilidad, ha sido el patrimonio de los entes económicos o sus expresiones de riqueza, en sus diversas manifestaciones y/o consecuencias. Millán (1992) comparte esta opinión al referirse al objeto de estudio de la contabilidad: "el patrimonio de la empresa y sus fenómenos son ante todo, las primeras fuentes de estudio de la contabilidad". El patrimonio del ente eco-

$7 \quad$ Zaá Mendez, José R. Conferencia ofrecida en I Simposio Internacional sobre las fronteras de la investigación y del conocimiento contable, Huancayo, Perú, Junio 2012. 
nómico posee una dinámica propia, es decir, sus incrementos y disminuciones, los cuales son consecuencia de los diferentes hechos económicos que lo afectan. Entiéndase por hecho "el fenómeno estudiado, lo que aparece ante nuestros sentidos, pero no es algo puesto por el sujeto, es decir, creado o manipulado, sino que es algo dado por el entorno, que no tiene ni esencia ni sustancia alguna, por lo que es lo mismo estudiar cosas orgánicas que inorgánicas" (Cappelleti, 1994). Bunge (1960) señala que "en todos los campos, la ciencia comienza estableciendo los hechos; esto requiere curiosidad impersonal, desconfianza por la opinión prevaleciente, y sensibilidad a la novedad". Por tanto, son los hechos económicos (transacciones económicas) que acontecen o que realizan los entes económicos los que afectan y le otorgan precisamente esta dinámica, constituyendo conjuntamente con el patrimonio, el objeto de estudio de la contabilidad.

Para Villarroya (s/f), el objeto de la contabilidad se orienta a la captación y representación de las transacciones que las empresas realizan con el mundo exterior, buscando llegar a un conocimiento lo más exacto posible de las mismas con la finalidad de determinar el resultado y la situación económico financiera de la empresa, es decir, la riqueza en un momento determinado y la renta de un período. Rodriguez Ariza (1985) menciona que el objeto es la realidad principalmente económica, tanto en su aspecto estático y dinámico. De la misma opinión es Gómez $\operatorname{Lopez}^{8}$ (s/f) al señalar que la contabilidad obtiene y suministra información del estado y evolución de determinadas realidades económicas...
Concretando sobre el objeto de la contabilidad, es cierto que existen muchas posturas filosóficas en relación a lo que es la realidad, diríamos que la contabilidad trabaja sobre una realidad social, ya que gran parte de los conceptos que emplea se refieren a aspectos que se desarrollan en el ámbito de las relaciones entre humanos y los grupos sociales que ellos conforman, como por ejemplo las ideas de propiedad, control, deudas y obligaciones, entre otras (Carrizo y León, 2007). Es decir, la contabilidad observará y captará hechos, reales en el plano de lo social, y los transformará en información útil de modo de representar la realidad económica de un ente, hecho que le permitirá controlarlo. Como se puede entender, la realidad está compuesta por una serie de hechos o fenómenos, pero el objeto de estudio de la disciplina contable son los hechos de carácter económico.

¿Cuál es el método de la disciplina contable?

No es sino hasta el siglo XIX en que se cuestiona el método denominado partida doble, el cual es un algoritmo matemático ${ }^{9}$ constituido por un elemento simbológico de representación de la realidad transaccional, propia de una sociedad mercantil de intercambios limitados. Este método, respondía a un sistema de representación económica, debido a que establece un medio de registración para cada ciclo económico (Fernández, 1977).

El método para la obtención del conocimiento denominado científico es un procedimiento riguroso, de orden lógico, cuyo propósito es demostrar el valor de verdad de ciertos enunciados. Augusto Comte fue

8 Gómez López (s/f). La ciencia contable: fundamentos científicos y metodológicos.

9 La contabilidad desde su creación como disciplina fue proclive a la acción repetitiva y mecánica de los haceres, en donde no existió una actividad cognoscitiva que vertebrara y dirigiera la construcción de estructuras conceptuales continuas, metodológicas, axiológicas y actitudinales ... 
quién trasladó el método científico inductivo de las ciencias físico-naturales a las ciencias sociales, desarrollando un modelo desde la perspectiva de la ciencia empírica experimental para alcanzar la percepción de los fenómenos naturales y sociales. Esta postura es conocida como positivismo y fue la que dio fuerza a los principios, proporcionó métodos y formas de operar en la contabilidad; pero que, a su vez la hizo parecer a la disciplina contable más como una técnica que como una ciencia. La idea inmersa en esta corriente es que se pueden utilizar los mismos métodos que se usan para estudiar un ser vivo, para proporcionar información y para revisar estos hechos o fenómenos. Su fundamentación en palabras de Cappelletti (1994) es sencilla: "lo único que se puede conocer es él hecho".

El profesor Cañibano (1996) al referirse al "método contable" señala que es "un conjunto de postulados y premisas subsidiarias que permite someter a observación la realidad económica, expresar en un lenguaje convenido los aspectos cualitativos y cuantitativos de dicha observación conforme a unas reglas que garantizan un determinado grado de objetividad, y procesar la información resultante siguiendo unos criterios que permitan obtener estados sintéticos que contengan agregados relevantes”. Millan (1992) en forma más precisa señala que los métodos o instrumentos de investigación de la contabilidad son: inductivos, deductivos, lógicoexperimentales y estadísticos.

El método contable es un proceso sistemático y lógico el cual comprende las siguientes fases: el análisis de las transacciones en aplicación del principio de dualidad, su cuantificación, es decir, su medición y valoración, su registro contable y por último la agregación Villarroya $(\mathrm{s} / \mathrm{f})^{10}$. Este proceso surge del raciocinio de la inducción (De la torre, 1991). La fase tal vez más importante que identificamos en cuanto a este proceso lógico es la cuantificación, por lo que Viloria y Casal (2007) señalan que el método fundamental de la contabilidad ha sido la cuantificación de todas las magnitudes contables, en detrimento de la cualificación o explicación amplia y profunda, todo ello como producto de la objetivización positivista.

Gómez López (s/f) desde una perspectiva más patrimonial precisa que la metodología específica desarrollada por la contabilidad para obtener y suministrar información del estado y evolución de determinadas realidades económicas se materializa en las funciones de captación, simbolización, medida, valoración, representación, coordinación, agregación, que junto a las de análisis e interpretación, y sirviéndose inicialmente de un proceso de inducción que posteriormente se torna en deductivo, permite la obtención de la realidad económica revelada, representativa, en términos contables, de una realidad que mediante una simple observación se presentaría inexpresiva y desordenada. En este proceso de representación de la realidad económica, se señala que acontece un fenómeno: la interrelación y conexión que se establece entre los distintos elementos que la componen dando lugar a una coordinación o conjunción armonizada de la distinta fenomenología captada, que completa al mencionado proceso de representación, que el profesor Calafell (1971) lo denomina: Proceso Metodológico Contable Integral, que está compuesto por dos subprocesos - el primero inductivo y el segundo deductivo - $y$ otro intermedio entre ambos. El primer subproceso se caracteriza por un razonamiento inductivo

10 VILlarROYA, L. Ma. Begoña, Manual de Contabilidad Financiera y analítica, Universidad de Valladolid, España, p. 66. 
que culmina con la obtención del Balance, y dentro de aquel se distinguen dos partes fundamentales: 1) Una de naturaleza científica, en la que se lleva a cabo la captación, simbolización, medida y valoración de la realidad económica. 2) Otra de naturaleza técnicoformal, que se ocupa de la representación, coordinación e integración de dicha realidad hasta llegar a la formulación del Balance de situación.

La utilización del método inductivo queda patente en cuanto que la Contabilidad parte de la observación de hechos, fenómenos o realidades particulares, hechos contables susceptibles de ser captados y representados, de tal forma que, aislándolos de otros, los capta, interpreta, mide y valora, a través del análisis pre-contable. Así, mediante el empleo de entes conceptuales adecuados, resultan susceptibles no sólo de la utilización de los distintos sistemas contables llegándose, por sucesivas agregaciones, a la formulación de diversos estados contables, síntesis de esa realidad económica estudiada y máximos exponentes de esta operatoria inductiva (Rodriguez Ariza, 1992). El segundo subproceso, por su parte, tiene lugar a partir del Balance y se caracteriza por un razonamiento deductivo que permitirá revelar la realidad económica en todos sus aspectos, constituyendo así el proceso de análisis contable. A su vez, en este subproceso se distinguen dos aspectos fundamentales: 1.- Análisis de la historia económica: se desprende de la Contabilidad, e implícitamente del Balance y documentos anexos. 2.- Análisis prospectivo o de expectativas: sirve de base en la toma de decisiones futuras sobre la gestión a realizar.

Además de ambos subprocesos, existe otro entre ellos que el citado autor denomina subproceso intermedio y que se ocupa de dos importantes problemáticas contables: 1) La verificación o constatación contable en su doble aspecto técnico contable y económico. 2) La consolidación o integración contable de varias situaciones económicas en una sola situación. Todo lo anterior debe tener como origen o punto de partida una organización contable, en la que se tengan presente todos los fines que pretenden ser alcanzados (Calafell, 1971).

Los últimos avances en investigación realizados sobre el saber contable, demuestran que existe una corriente doctrinaria que aplica el método científico deductivo en el estudio de la contabilidad y encara los problemas de la misma incluyendo la utilización de hipótesis, leyes y teorías. El método deductivo como método de investigación con sustento científico puede ser aplicado para establecer la ubicación, el dominio y los supuestos básicos de una disciplina (García Fronti, 2006) ${ }^{11}$.

¿Posee la disciplina contable capacidad predictiva?

La función predictiva de la disciplina contable se evidencia desde hace muchísimos años. El profesor Joseph Vlaemminck en su obra "HISTORIA Y DOCTRINA DE LA CONTABILIDAD" menciona que en los registros de los MASSARI de GENOVA (1340) llevados a la veneciana aparecen cuentas de presupuesto. Luego cita al monje benedictino ANGELO PIETRA como el primer autor que se preocupa de la revisión de ingresos y gastos (siglo XVI) y a DOVICO FIORI como el autor que habla de la utilidad de los presupuestos (1636), que no son otra cosa que manifestaciones de la función predictiva contable.

En el actual contexto de los negocios se requiere que la información para la toma de decisiones se concentre fundamentalmente

11 García Fronti, Inés (2006). Versión actualizada y completada del trabajo publicado en Metodología de la Investigación Contable, Errepar, 2006, Buenos Aires. 
en obtener la mayor cantidad de datos posibles que permitan predecir los resultados. Los estados contables deberían brindar la información necesaria a fin de que los usuarios externos -los más afectados por la insuficiencia de la información histórica- puedan evaluar aspectos tales como: riesgo inherente a su inversión, la probabilidad de que la empresa pague dividendos, alternativas de comprar, vender o retener sus participaciones, capacidad de la empresa de afrontar sus obligaciones, etc. En general, estas decisiones están asociadas con las expectativas de la empresa de generar flujos de fondos en el futuro (Suardi, Nannini y otros, 2004)

La capacidad de predicción de una ciencia, requiere necesariamente del mecanismo de la deducción del pensamiento lógico que se da a través de un proceso y consiste en que dadas determinadas proposiciones tomadas como premisas, consideradas por definición, hipotéticamente como verdaderas; de ellas se saca la verdad que entrañan; y, en razón de su relación estructural (o formal) con otra (s) proposición(es) llamada conclusión, dicha conclusión es necesariamente verdadera, porque su verdad le es transmitida de sus premisas.

El conocimiento científico se encarga de dar respuesta a todas aquellas cuestiones que preocupan al hombre, además de este fin teleológico, puede tener otros, como un fin descriptivo: descripción de los fenómenos mediante una teoría, predictivo: conocer la ocurrencia de un evento, e incluso puede orientar la adopción de decisiones en la resolución de problemas prácticos, es decir, una función prescriptiva o de control (Villarroya, $\mathrm{s} / \mathrm{f}$ ). La predicción contable encuentra su antecedente en la explicación contable, no solo porque al igual que la explicación encuentra su antecedente en la descripción, sino porque la explicación contable conduce siempre hacia el futuro. Nadie en Contabilidad busca conocer los resultados de la gestión empresarial (léase variación o modificación del Patrimonio) por el solo hecho de conocerlo, sino que el hombre de ciencia explica como acontecieron los hechos económicos, establece la relación causa-efecto, va en busca de las causas en la explicación, en buena cuenta responde con calidad a la interrogante científica ¿por qué aconteció así?

La predicción implica "una traslación temporal de la explicación establecida, desde un intervalo de tiempo pasado y conocido, hasta otro intervalo de tiempo futuro y por conocer" (Eli De Gortari, s/f). La predicción responde a las preguntas ¿qué sucederá?, ¿cómo sucederá? si se dan determinadas premisas. Cuando esa(s) respuesta(s) se $\mathrm{da}(\mathrm{n})$ el científico contable encuentra sus premisas, hipotéticamente verdaderas, que van a sustentar su acción predictiva (léase su actividad futura) en una realidad aún no conocida, sino por conocer.

La contabilidad formulada sobre el principio de empresa en marcha, es fundamentalmente futurista. El pensamiento moderno, lógico-científico no busca conocer la realidad y explicar esta por el solo hecho de conocer leyes de su desarrollo, sino fundamentalmente para controlar la realidad, modificarla o prevenirla, en sumo, acciones futuras que son, hoy por hoy, el quehacer primordial del hombre de ciencia, y de la disciplina que abrace por lo que se puede afirmar que si un área del conocimiento humano (disciplina científica) no cumple esta función, corre el riesgo de perder vigencia $y / o$ de desaparecer.

\subsubsection{Evidencias de la capacidad predictiva de la contabilidad}

La dinámica del mundo de hoy requiere que las decisiones se tomen a cada minuto sobre la información presente y del futuro, por tan- 
to, se hace evidente la necesidad de contar con información financiera proyectada. El propósito fundamental de la información proyectada sería la de proporcionar a los usuarios de la misma, previsiones razonables y fundadas de los equilibrios económico y financiero de la entidad, de manera que dichos usuarios puedan tomar sus decisiones basándose en estimaciones racionales, reduciendo, en la medida de lo posible, el riesgo que conllevan estos, si no se dispone de este tipo de información (Suardi y otros, 2004).
La ciencia contable cuenta con modelos predictivos. Los modelos predictivos se pueden utilizar para controlar la evolución de la empresa al comparar lo previsto con la realidad, permitiendo tomar decisiones a tiempo para intentar incidir sobre el futuro y hacerlo más favorable. Con la predicción de ciertos hechos se pueden tomar medidas correctivas a partir de las desviaciones detectadas antes que sea demasiado tarde. A continuación de enuncian los modelos predictivos.

\begin{tabular}{|c|c|}
\hline MODELO CONTABLE & PROPOSITO \\
\hline Presupuesto & $\begin{array}{l}\text { Es utilizado como elemento de control de gasto } \\
\text { tanto en el sector público como en él privado, } \\
\text { como base en la necesidad formulada por } \\
\text { funcionarios cuya labor es presupuestar con la } \\
\text { finalidad de garantizar el eficiente funcionamiento, } \\
\text { sostenimiento y liderazgo en las actividades } \\
\text { económicas de las organizaciones. }\end{array}$ \\
\hline Las Reservas & $\begin{array}{l}\text { Las Reservas sirven para proveer fondos } \\
\text { destinados a cubrir compromisos que en el futuro } \\
\text { debe afrontar la empresa como consecuencias de } \\
\text { operaciones del ejercicio y/o contingencias que } \\
\text { pueden ocurrir. }\end{array}$ \\
\hline EI Flujo de caja proyectado & $\begin{array}{l}\text { Con este se elaboran las estrategias financieras, } \\
\text { permitiéndole a la gerencia financiera saber con } \\
\text { anticipación las medidas correctivas que deberá } \\
\text { adoptar en el transcurso del ejercicio económico } \\
\text { para el cual fue diseñado el proyecto }\end{array}$ \\
\hline Los Estados Financieros proyectados & $\begin{array}{l}\text { Son cuadros que representan el Estado de } \\
\text { Situación Financiera y Económico (Estado de } \\
\text { Resultado Integral) de una empresa a una fecha o } \\
\text { periodos futuros, basándose en cálculos } \\
\text { estimados o transacciones que aún no se han } \\
\text { realizado. Son estados que se acompañan } \\
\text { frecuentemente con un presupuesto. }\end{array}$ \\
\hline Planeamiento Tributario & $\begin{array}{l}\text { Es el procedimiento de buscar estrategias fiscales } \\
\text { en el marco de las disposiciones legales vigentes } \\
\text { para minimizar la carga tributaria de las empresas } \\
\text { y para tener un conocimiento anticipado de las } \\
\text { futuras obligaciones tributarias. }\end{array}$ \\
\hline Los Modelos de predicción de quiebra & $\begin{array}{l}\text { Estos modelos pueden ser utilizados para medir la } \\
\text { capacidad de información contable para predecir } \\
\text { la futura solvencia de la empresa. }\end{array}$ \\
\hline El Balance Scorecard & $\begin{array}{l}\text { Herramienta que permite implementar la estrategia } \\
\text { y la misión de una empresa a partir de un conjunto } \\
\text { de medidas de actuación. Pone énfasis en la } \\
\text { consecución de objetivos financieros, e incluye los } \\
\text { inductores de actuación futura para el logro de } \\
\text { esos objetivos. Proporciona una estructura para } \\
\text { transformar la estrategia en acción. }\end{array}$ \\
\hline Modelos de factores de Riesgo & $\begin{array}{l}\text { Debido al grado de incertidumbre, se deben tener } \\
\text { en cuenta los factores que pueden influir para que } \\
\text { lo estimado no se cumpla. Dichos factores pueden } \\
\text { ser: factores internos y factores externos. }\end{array}$ \\
\hline
\end{tabular}




\section{DISCUSION}

Aunque no han faltado autores que han negado la autonomía científica de la disciplina contable, tales como Gino Zappa (1929), Mardones (1991), y otros. Mardones por ejemplo, precisa que existe una polémica sobre el estatuto de cientificidad de la contabilidad y que ello se origina desde la aparición de las diversas disciplinas que se acogen a la categoría de ciencias económicas y administrativas. Sin embargo, sus razonamientos parecen haber quedado totalmente falsados. Es así que Ballestero (1983) menciona que la contabilidad que ha superado las "teorías" pre-científicas de la escuela italiana, ha desarrollado axiomas y dispone de métodos operativos de sofisticación intermedia, por lo que se está aproximando notablemente al estatus de ciencia.

La contabilidad ha seguido la suerte de las ciencias naturales, dado que lo predominante de estas ha sido el desarrollo acumulativo de las herramientas matemáticas y lógicas, llegando a lo que se denomina "reduccionismo" o crisis paradigmática de la contabilidad (Zá, 2012), ya que actualmente la disciplina contable no responde a las nuevas exigencias del entorno, para dejar de presentar cifras frías sin contexto y en su lugar, tomar una posición frente a la necesidad de registrar y valorar los recursos naturales, cuantificar los costos sociales de la educación o la salud, etc.

El término paradigma, introducido por Kuhn, el cual no tiene una definición unívoca (Barker, 1995), pero constituye la característica que distingue la ciencia -ciencias maduras- de la no ciencia. Las ciencias en formación, no tienen los paradigmas como una constante (Montes, 2006). Al contrario, ellas poseen múltiples visiones y posiciones que, a manera de interpretación y elevadas a la categoría de teorías, buscan se les acepte como alternativa válida en el proceso de descripción, explicación, predicción.

Algunos autores han profundizado en torno a la fundamentación de la Contabilidad como ciencia basándose en los caracteres que, según la lógica, deben reunir unos conocimientos para ser considerados como científicos, estudiando el objeto material, el objeto formal y el fin de la ciencia

contable (Fernández Pirla, Calafell Castelló, 1963). Otros han utilizado la Axiomática de las ciencias formales y han constituido sistemas de axiomas, teoremas, postulados y definiciones a las cuales someten la realidad económica con objeto de ser tratada con rigor científico y de enunciar los principios y normas de la ciencia de la Contabilidad (Mattessich, Holzer). Por último, en la actualidad, existe un grupo de autores que aplican a la demostración científica de la Contabilidad los principios de la teoría de conjuntos y de la lógica simbólica, tendiendo a una teoría formal de la Contabilidad (Devine, Ijiri, Mattessich)".

Debido a que no todas la ciencias pueden experimentar y considerando que los resultados experimentales son pocas veces interpretados de una sola manera, la ciencia fáctica es empírica en el sentido de que la comprobación de sus hipótesis involucra la experiencia (una de las reglas del método científico); pero no necesariamente experimental, ya que la aplicación de esta regla depende del tipo de objeto, del tipo de la hipótesis en cuestión y de los medios disponibles. Por esta razón, se señala que se necesita una multitud de técnicas de verificación empírica. La verificabilidad es la esencia del conocimiento científico objetivo (Bunge, 1960). La disciplina contable se refiere a hechos plenamente verificables. 
Desde la perspectiva epistemológica clásica $^{12}$, las disciplinas científicas se caracterizan, por la identificación de unos elementos propios mínimos: el Objeto sobre el que la forma de conocimiento trata; el método por el cual es abordado tal objeto de la realidad; las teorías que se construyen a partir del paulatino descubrimiento de la misma realidad; y el sujeto mismo que conoce (Serrano, 1996; Pág. 124). Esta propuesta se centra en identificar estos elementos que otorgan el estatus de ciencia a una disciplina, los cuales se han identificado a lo largo de la evolución de la definición de ciencia. Esta característica que exigimos es la capacidad predictiva que toda disciplina con estatus de ciencia debe poseer.

La contabilidad no es sólo un saber puramente lógico-matemático, ni se reduce a la matematización de la facticidad objetiva, su fuente interpretativa se encuentra en la realidad social o mundo extra-subjetivo. Esta realidad social es un todo integrado, que comporta un carácter dual en términos de facticidad objetiva y significado subjetivo, que la hace "sui generis" (Martinez Pino, $\mathrm{s} / \mathrm{f}$ ). Además, la concepción de la contabilidad como ciencia social no le impide poseer cierto grado de matematización, debido a lo que señala Horkheimer (1995; Pág. 23) "las disciplinas sociales con el mayor nivel de manejo matemático en su formalización y validación conceptual (el caso de la economía), adquieren el calificativo de ser ciencias sociales maduras". Entonces, bajo esta consideración incluso la disciplina contable con su actual contenido matemático que no le impide ser redefinida como ciencia social y sin tener ese "mayor nivel matemático" que se precisa, estaría calificando como ciencia, pero sin alcanzar tal vez el nivel de ciencia madura. Además, la formulación matemática, deseable como es, no es una condición indispensable para que el conocimiento sea científico; lo que caracteriza el conocimiento científico es la exactitud en un sentido general antes que la exactitud numérica o métrica, la que es inútil si media la vaguedad conceptual (Bunge, 1960).

Las primeras manifestaciones de nuestra disciplina se adscribieron ha ámbitos próximos a las matemáticas y, en especial a la aritmética comercial (Tua Pereda, 1995). Chaves da Silva (2007) nos ilustra que el objeto de estudio de la disciplina contable siempre estuvo situado alrededor del patrimonio de la entidad o la acumulación de la riqueza. Esta riqueza de los entes económicos, en sus diversas manifestaciones, se expresa a través de su patrimonio, el cual posee una dinámica propia, es decir, sus incrementos y disminuciones, los cuales son consecuencia de los diferentes hechos económicos que lo afectan. Por tanto, son los hechos económicos (transacciones económicas) que acontecen o que realizan los entes económicos los que afectan y otorgan la dinámica de la riqueza, precisamente, el objeto de estudio de la contabilidad. En un lenguaje más patrimonialista, Gómez López (s/f) precisa que el objeto de la contabilidad es informar el estado y evolución de determinada realidad económica.

La identificación del objeto de estudio de la disciplina contable que señalamos, es coincidente con lo expresado por Bunge (1960) "en todos los campos, la ciencia comienza estableciéndose los hechos...” y, por Cappelletti (1994) al mencionar que por "hecho se entiende el fenómeno estudiado, lo que aparece ante nuestros sentidos, pero no es algo

12 Nos referimos aquí a la filosofía de las ciencias y del conocimiento, desde Aristóteles, pasando por descartes y llegando a los empiristas y positivistas. En Briones Guillermo. La ciencia y la modernidad. Editorial Ariel. México 1964. 
puesto por el sujeto, es decir, creado o manipulado, sino que es algo dado por el entorno, que no tiene ni esencia ni sustancia alguna, por lo que es lo mismo estudiar cosas orgánicas que inorgánicas". Villarroya (s/f) de una forma más precisa expresa que el objeto de la contabilidad se orienta a la captación y representación de las transacciones que las empresas realizan con el mundo exterior.

El término "método contable" se refiere al conjunto de postulados y premisas que después de observar la realidad económica nos permite comunicarla a través de los estados contables (Villarroya, $\mathrm{s} / \mathrm{f})^{13}$, que se formulan, haciendo uso de un proceso sistemático y lógico. La contabilidad se sirve de un proceso inductivo que inicialmente se materializa en las funciones de captación, simbolización, medida, valoración, representación, coordinación, agregación, que junto a las de análisis e interpretación, tornándose después en deductivo, para permitir la obtención de la realidad (Gomez, s/f). En este mismo sentido se expresan Calafell (1971), al señalar que el proceso metodológico contable integral, está compuesto por dos subprocesos: el inductivo y el deductivo y Rodriguez Ariza (1992), al señalar que "la utilización del método inductivo queda patente en cuanto que la Contabilidad parte de la observación de hechos, fenómenos o realidades particulares, hechos contables susceptibles de ser captados y representados, de tal forma que, aislándolos de otros, los capta, interpreta, mide y valora, a través del análisis pre-contable...."

Los últimos avances en investigación realizados sobre el saber contable, demuestran que existe una corriente doctrinaria que aplica el método científico (Método deductivo) en el estudio de la contabilidad y encara los problemas de la misma incluyendo la utili- zación de hipótesis, leyes y teorías. Independientemente de quienes precisan sus opiniones sobre el Método Contable, todos ellos coinciden en señalar que este es aplicado con la finalidad de someter a observación la realidad económica de los entes, la cual se expresa en su patrimonio y su dinámica, originada por las transacciones o hechos económicos, reafirmando nuestra opinión sobre el objeto de estudio. Para nosotros, después de la revisión de la literatura, es claro que el método de la ciencia contable descrito aquí, es el método Inductivo-Deductivo.

Con relación a la capacidad predictiva contable, debemos tener presente que esta disciplina elabora información sobre la base del principio de empresa en marcha, es decir, se orienta hacia el futuro y no sólo busca conocer los resultados de la gestión empresarial por el hecho de conocerlo, sino que el hombre de ciencia explica como acontecieron los hechos económicos, establece la relación causa-efecto, va en busca de las causas en la explicación, en buena cuenta responde con calidad a la interrogante científica ¿por qué aconteció así? La predicción en cambio, responde a las preguntas ¿qué sucederá?, ¿cómo sucederá? si se dan determinadas premisas. Cuando esa(s) respuesta(s) se da(n) el científico contable encuentra sus premisas, hipotéticamente verdaderas, que van a sustentar su acción predictiva (léase su actividad futura) en una realidad aún no conocida, sino por conocer.

\section{CONCLUSIONES}

1. El paradigma contable actual exclusivamente cuantitativo, se ha desequilibrado al otorgar mayor peso a los métodos y a las normas, que a la propia teoría; habiendo

13 VILLARROYA, L. Ma. Begoña, Manual de Contabilidad Financiera y analítica, Universidad de Valladolid, España, p. 66. 
sido útil al desarrollo de la disciplina contable hasta mediados del siglo pasado. El camino para superar esta problemática contable y proveernos de soluciones que permitan disminuir el grado de incertidumbre de los usuarios de la información financiera es la investigación científica. La contabilidad es una realidad cambiante que procura estudiar científicamente esa realidad. El conocimiento contable es racional al formular juicios y razonamientos coherentes entre sus enunciados. Sin embargo, atendiendo a los principios y no al desarrollo formal de las ciencias, los cuales pueden racionales o experimentales, sostenemos que es una ciencia aplicada y experimental y se encuentra en proceso de maduración, debido a que no existe un paradigma predominante, siendo una ciencia multi-paradigmática.

2. El objeto de estudio de la contabilidad es la realidad económica, la cual se expresa a través del patrimonio y su dinámica. El método contable siendo inductivo-deductivo, está compuesto por el conjunto de postulados y premisas que después de observar la realidad nos permite comunicarla, siguiendo para ello y proceso sistemático y lógico. Las fases que comprende el método contable son: el análisis de las transacciones en aplicación del principio de dualidad, su cuantificación, es decir, su medición y valoración, su registro contable y por último la agregación. El método contable es Inductivo hasta culminar el Balance y deductivo porque partiendo del Balance permite revelar la realidad económica en todos sus aspectos, constituyendo así el proceso de análisis contable. Finalmente, la información contable al ser elaborada bajo el principio de empresa en marcha, es de corte futurista o prospectiva y hace posible el análisis de expectativas, la elaboración de los presupuestos, el establecimiento de reservas, el análisis de riesgo, todas ellas herramien- tas base para la toma de decisiones futuras. Del mismo modo, las políticas contables y la uniformidad de procedimientos, permite la elaboración de varios modelos tales como: Estados Financieros y Estados de Flujo de caja proyectados, el planeamiento tributario, predicción de quiebra, la construcción de herramientas para la gestión y control como el Balance ScoreCard, evidenciando de esta forma la capacidad predictiva de la disciplina contable.

\section{REFERENCIAS BIBLIOGRÁFICAS}

1. BELLO REGUERA, Eduardo (2006). El Discurso del Método: Estudio preliminar, Edit. Tecnos, Sexta edición, Madrid, España.

2. BUNGE, Mario. La ciencia: su método y su filosofía. Ariel, Buenos Aires, Argentina, 1960.

3. BUNGE, Mario. Epistemologia, Barcelona, Ariel 1980.

4. BURBANO RUIZ, JORGE E. Y ORTIZ GOMEZ, ALBERTO (1988). Presupuestos: enfoque moderno de planeación y control de recursos. Editorial McGraw-Hill, segunda edición, Santafé de Bogotá, Colombia.

5. CALAFELL CASTELLÓ, A. (1971): Apuntes de Introducción a la Contabilidad, Departamento de Contabilidad de la Facultad de Ciencias Económicas y Empresariales, Universidad Autónoma, Madrid, Curso 1971-1972.

6. CAÑIBANO CALVO, L. (1996): Contabilidad. Análisis de la realidad económica, Madrid: Ed. Pirámide.

7. CAPPELLETTI, Angel (1994). Positivismo y evolucionismo en Venezuela. Caracas: FACES-UCV. 
8. CARRIZO, Walter y Silvia LEON (2007), ¿Qué realidad representa la contabilidad? Revista Pecvnia, 5, pp. 17-27. Universidad nacional de la Patagonia, Provincia de Chubut, Argentina.

9. CASAL y VILORIA (2002). La corriente positivista y su influencia en la ciencia contable. Actualidad Contable FACES, año 5, $\mathrm{N}^{\circ}$ 05, Enero Junio, 2002.

10. CASAL y VILORIA (2007). La ciencia contable, su historia, filosofía, evolución y su producto. Actualidad Contable FACES, Venezuela. Año 10, $\mathrm{N}^{\circ} 15$, JulioDiciembre 2007.

11. CAVADA, Daniel (2004). Ciencias Naturales y Ciencias Sociales: Un debate sobre su acercamiento inter-paradigmático, Tesis para optar el grado de sociólogo, Chile.

12. De ANDRADE MARTINS, Gilberto. Hablando de Teorías y Modelos Contables. Actualidad, Año 9, $\mathrm{N}^{\circ}$ 13, Contable FACES, Julio-Diciembre 2006, Mérida, Venezuela.

13. DOMINGUEZ G., Silvia. "Objeto de estudio en la investigación: diversas aproximaciones", Revista de Educación y Desarrollo, $\mathrm{N}^{\circ}$ 7, Octubre Diciembre, 2007.

14. ECHEVARRIA, J. (2002). Ciencia y Valores. Barcelona: Destinos.

15. EYSSAUTIER de la MORA, Maurice (2002). Metodología de la Investigación: Desarrollo de la Inteligencia. ECAFSA Thompson Learning, 4ta edición, México.

16. FERNANDEZ PIRLA, José María (1977). Teoría económica de la contabilidad-Introducción contable al estudio de la economía. Ediciones ICE, Novena edición, Madrid-España.
17. GALLEGO BADILLO, Rómulo (1996). Discurso constructivista sobre las ciencias experimentales -una concepción actual del conocimiento científico. Editorial Mesa Redonda-Magisterio. Santafé de Bogotá.

18. GOMEZ LOPEZ, Roberto (s/f). La ciencia contable: fundamentos científicos y metodológicos. Universidad Nacional de educación de Málaga, España.

19. GOMEZ VILLEGAS, Mauricio (2003). Comentarios sobre el discurso científico y los determinantes morales, FENECOP, Colombia.

20. HEIDEGGER, M. "La época de la imagen del mundo", en sendas perdidas. Losada, Buenos Aires, 1960.

21. HORNGREN y Otros(1997), Contabilidad, editorial Pearson Education, Tercera edición, México, p. 4.

22. KAPLAN, ROBERT S. Y NORTON, DAVID P. (2000). Cuadro de Mando Integral: The Balanced Scorecard. Gestión 2000, segunda edición, Barcelona, España.

23. MARCOS, Alfredo (2010). Ciencia y Acción. Edit. Fondo de cultura económica, primera edición, México.

24. MARDONES, J. (1991). Filosofía de las ciencias humanas y sociales. España: Anthropos Editorial del Hombre.

25. MARIAS, Julián (1971), Historia de la filosofía, Edit. Revista de Occidente, S.A., $23^{a}$ edición, Madrid, España, p. 240-242.

26. MAZZI HUAYCUCHO, Víctor (2006); Epistemología - selección de lecturas, Edit. San Marcos, Lima, Perú.

27. MEJIA SOTO, Eutimio (2011). Introducción al pensamiento Contable de García Casella. Edit. Optigraf Ltda, Armenia, Quindio, Colombia. 
28. MILLAN PUENTES, Regulo (1992). La contabilidad como ciencia. Edit. Félix Rodriguez Ltda, Santa Fé de Bogotá, Colombia.

29. MONTES S., Carlos A., OTO M., E. Y VALENCIA S., John J. (2006). Paradigmas en Contabilidad, Edit. Artes Graficas del Valle, Cali, Colombia.

30. MORA ENGUIDANOS, ARACELI (1995). Utilidad de los modelos de predicción de la crisis empresarial. Revista española de financiación y contabilidad Vol. XXIV, N 83, abril-junio 1995, pp. 281-300.

31. MORIN, Edgar y otros (2003), Educar en la era planetaria, Capítulo I: El Método, Editorial Gedisa.

32. MOSTERIN, Jesús (2011). Filosofía y ciencia: un continuo, Fondo Editorial: Universidad Inca Garcilaso de la Vega, Lima, Perú.

33. PEREZ TAMAYO, Ruy (2010). ¿Existe el método científico?, Edit. Fondo de cultura económica, Quinta reimpresión de la tercera edición, México.

34. ROJAS OSORIO, Carlos (2001). Invitación a la Filosofía de la Ciencia, Humacao, Brasil.

35. SOLOMON, VARGO, SCHROEDER, Principios de Contabilidad, Edit. Harla, 1ra edición en español, México.

36. SUARDI, NANNINI, CARAZAY y otros (2004). Los nuevos desafíos de la contabilidad. Novenas Jornadas de investigación en la facultad de ciencias económicas y estadística. Buenos Aires.
37. TAMAYO Y TAMAYO, Mario (1999). El proceso de la investigación científica, Editorial Limusa, Duodécima reimpresión de la tercera edición, México.

38. TUA PEREDA, Jorge (1995). Lecturas de teoría e investigación contable. Centro Interamericano Jurídico-Financiero. Ediciones Graficas Ltda. Medellin-Colombia.

39. UGARTE SILVA, Alfonso. La realidad de la contabilidad. S/F

40. VILLARROYA L., Ma. Begoña, Manual de Contabilidad Financiera y analítica, Universidad de Valladolid, España.

41. WELLS, M.C. (1976): A Revolution in Accounting Thought, The Accounting Review, Vol. LI, no 3, July.

42. WIRTH (1999). La realidad de la contabilidad. Tesis Doctoral.

43. ZAA MENDEZ, José Rafael (2012). Teoría Neopatrimonialista de la Contabilidad: Filosofía y modelos de desarrollo científico de la contabildad. Huancayo, Junín, Perú.

44. ZAMBRANO, María. Notas de un Método, Mandadori, Madrid 1989.

45. Revista electrónica Tercera Cultura: "Cuando no podemos predecir", 23-052011. http://www.terceracultura.net/ tc/?p=3134

46. Rodríguez de Rivera, José: www.robertexto.com/archivo5/racion_critico.htm

47. http://cybertesis.upnorte.edu.pe/ upnorte/2003/zelada_aw/xhtml/ TH.3.xml 